\title{
How Daphnia copes with excess carbon in its food
}

Received: 26 July 2002 / Accepted: 31 March 2003 / Published online: 19 June 2003

(C) Springer-Verlag 2003

\begin{abstract}
Animals that maintain near homeostatic elemental ratios may get rid of excess ingested elements from their food in different ways. C regulation was studied in juveniles of Daphnia magna feeding on two Selenastrum capricornutum cultures contrasting in $\mathrm{P}$ content (400 and $80 \mathrm{C}: \mathrm{P}$ atomic ratios). Both cultures were labelled with ${ }^{14} \mathrm{C}$ in order to measure Daphnia ingestion and assimilation rates. No significant difference in ingestion rates was observed between P-low and P-rich food, whereas the net assimilation of ${ }^{14} \mathrm{C}$ was higher in the treatment with P-rich algae. Some Daphnia were also homogeneously labelled over 5 days on radioactive algae to estimate respiration rates and excretion rates of dissolved organic $\mathrm{C}$ (DOC). The respiration rate for Daphnia fed with high C:P algae (38.7\% of body C day $^{-1}$ ) was significantly higher than for those feeding on low C:P algae $\left(25.3 \%\right.$ of body $\left.\mathrm{C} \mathrm{day}^{-1}\right)$. The DOC excretion rate was also higher when animals were fed on P-low algae $\left(13.4 \%\right.$ of body $\mathrm{C}$ day $\left.^{-1}\right)$ than on P-rich algae $(5.7 \%$ of body $\mathrm{C}$ day $\left.^{-1}\right)$. When corrected for respiratory losses, total assimilation of $\mathrm{C}$ did not differ significantly between treatments (around $60 \%$ of body $\mathrm{C}_{\text {day }^{-1}}$ ). Judging from these experiments, D. magna can maintain its stoichiometric balance when feeding on unbalanced diets (high $\mathrm{C}: \mathrm{P})$ primarily by disposing of excess dietary $\mathrm{C}$ via respiration and excretion of DOC.
\end{abstract}

Keywords Assimilation - Carbon:phosphorus ratios . Dissolved organic carbon excretion - Homeostasis . Respiration

F. Darchambeau

URBO-Laboratory of Freshwater Ecology,

Facultés Universitaires Notre-Dame de la Paix,

Rue de Bruxelles 61, 5000 Namur, Belgium

P. J. Faerøvig · D. O. Hessen $(\bowtie)$

Department of Biology,

University of Oslo,

P.O. Box 1027 Blindern, 0316 Oslo, Norway

e-mail: dag.hessen@bio.uio.no

Fax: +47 22854438

\section{Introduction}

The highly variable nutrient stoichiometry in autotrophs may be a challenge for grazers that have a tighter elemental regulation. There is commonly a mismatch in elemental ratios between requirements and supplies (Hessen 1992; Urabe and Watanabe 1992; Elser et al. 2000). In daphniids, which commonly are the most important grazers in lakes, $\mathrm{P}$ requirement for somatic growth is high whereas freshwater algae are frequently $\mathrm{P}$ deficient (Hessen 1992; Sterner 1993). Phytoplankton in lakes often show very high C:P ratios in comparison with consumer C:P ratios (Elser and Hassett 1994) and this could lead to P limitation of daphniid growth and lower food chain production (Sterner and Hessen 1994; Hessen 1997; Sterner et al. 1998; Hessen and Faafeng 2000). Two parallel physiological adaptations which may be used by animals to cope with this excess dietary $\mathrm{C}$ have been suggested: (1) improvement of the assimilation of the limiting element, and (2) reduced intake, storage or disposal of the element in excess. There are three potential physiological solutions to the problem of excess C in a herbivore's diet (Sterner and Hessen 1994). First, P-rich animals could reduce the $\mathrm{C}$ assimilation efficiency across the gut, while maintaining a high $\mathrm{P}$ assimilation efficiency. Or, if assimilated, excess $\mathrm{C}$ may be stored internally in $\mathrm{C}$-rich compounds like lipids. Finally, assimilated $\mathrm{C}$ may be disposed of through respiration or extracellular release of organic $\mathrm{C}$ compounds.

The first hypothesis, a reduction of $\mathrm{C}$ assimilation efficiency, was advocated in a study by DeMott et al. (1998) on Daphnia magna. They fed animals with a mixture of P-sufficient and P-deficient Scenedesmus, and observed a decrease of $30 \%$ in $\mathrm{C}$ assimilation efficiency while $\mathrm{P}$ assimilation efficiency remained constant when the atomic food C:P ratio increased from 80 to 164 . The enzymatic responses at the basis of this regulation have not been explored. The second hypothesis, storage of excess $\mathrm{C}$, does not seem to be very realistic in fastgrowing homeostatic organisms (i.e. during juvenile development). Feeding zooplankters with P-enriched 
seston did not alter the P:dry weight (DW) ratio of Daphnia longispina and Bosmina coregoni (Andersen and Hessen 1991). Still the use of adipose tissue for energy storage is widespread among marine copepods (Båmstedt 1986), and also Daphnia may display visible stores of internal lipids in the last juvenile instar and in adults (Tessier and Goulden 1982; Goulden and Place 1990). Yet, it is not clear how this would affect body C:P ratios. Lipid accumulation in Daphnia could be a sign of a Pdeficient diet (Groeger et al. 1991; Sterner et al. 1992; Sterner 1993). Still the rather tight homeostatic control in Daphnia, unless dietary C:P is extremely high, suggests that $\mathrm{C}$ storage is not very pronounced. DeMott et al. (1998) observed marked declines in P:DW when feeding D. magna with algae with very high C:P ratio (920). These findings are probably more likely to originate from a $\mathrm{P}$ deficiency in body tissue than from an accumulation of $\mathrm{C}$.

The third hypothesis has been less well explored. The ability of zooplankters to modify their respiratory rate or dissolved organic $\mathrm{C}$ (DOC) excretion rate when facing low food quality can induce relevant impacts on secondary production and elemental $\mathrm{C}$ pathways in ecosystems (Sterner 1997). More precisely, release of DOC vs. $\mathrm{CO}_{2}$ might have marked consequences on the transfer of algal $\mathrm{C}$ to the microbial loop and the $\mathrm{CO}_{2}$ balance of the system. Release of DOC through grazing has been well studied since Lampert's (1978) seminal works (e.g. Copping and Lorenzen 1980; Olsen et al. 1986; Richardot et al. 2001) and seems to originate from three different sources: (1) losses of organic substrates from prey during handling and feeding ("sloppy feeding"), (2) leakage of DOC from the faeces, and (3) actual secretion of DOC by the animal. Studies on the impact of grazing on DOC and bacterial uptake do not commonly distinguish between these three sources, and the contribution of each source to total flux is still unclear. Sloppy feeding cannot be element specific. We can assume that in this process all elements are lost in a proportion equivalent to that in the original food. So sloppy feeding cannot be used as a process for homeostatic regulation. Release from faeces is linked to the regulation of assimilation efficiency. If less $\mathrm{C}$ is assimilated from food during transfer in the gut, more C will potentially be lost via faeces. Direct excretion of DOC (Gardner and Paffenhöfer 1982) could be an interesting way for daphniids to get rid of previously assimilated C-rich, P-poor macromolecules like proteins (Elser et al. 1996), but this process has never been quantitatively studied. Likewise, although suggested by Plath and Boersma (2001), the ability of daphniids to increase their respiration rates when feeding on C-rich algae has never been studied directly. Thus Sterner's (1997) humorous remark: "the animal doing extra work in its environment (swimming?) in order to maintain its homeostasis" is still largely undebated, although studies by Sterner himself (Sterner et al. 1993) indicate, in fact, that strongly P-deprived animals become notoriously sluggish. This response will depend not only on the access to $\mathrm{P}$ relative to $\mathrm{C}$, but also on the absolute availability of $\mathrm{P}$ for the animals.

In this study we attempted, by use of tracer experiments, to reveal the impact of a food-quality deficiency on ingestion, C assimilation, respiration and DOC excretion rates in Daphnia magna Straus.

\section{Materials and methods}

Algae

The green alga Selenastrum capricornutum Printz was grown in the same continuous culture system as described by Hessen et al. (2002). Cultures were run in COMBO medium (Kilham et al. 1998) with a dilution rate of 0.4 day $^{-1}$ under two $\mathrm{P}$ regimes: $50 \mu \mathrm{M}(\mathrm{P}$ saturation) and $2.5 \mu \mathrm{M}$ (P limitation). Both received a nominal light intensity of $70 \mu \mathrm{mol}$ quanta $\mathrm{m}^{-2} \mathrm{~s}^{-1}$. These two regimes, referred to respectively as high quality (HQ) and low quality (LQ) treatments, supplied algae with C:P ratios nearing respectively 80 and 400 (atomic values). Selenastrum cultures were allowed to grow for 2 weeks before the start of labelling and experiments with Daphnia, in order to obtain a stable level of cell numbers, cell volumes and particulate C, N and P. For Daphnia incubations and labelling, the algae were labelled with $7.3 \mathrm{MBq} \mathrm{NaH}{ }^{14} \mathrm{CO}_{3} \mathrm{I}^{-1}$ for a 2-day period in batch cultures. Medium and radioactive substrate were replaced twice a day at the same dilution rate that algae underwent in the continuous system. This procedure provided saturating, uniform algal labelling with algal $\mathrm{C}: \mathrm{P}$ ratios similar to those observed in the algae taken directly from the continuous cultures (data not shown). High specific radioactivities were obtained (ca. 25,000 and $15,000 \mathrm{dpm} \mu \mathrm{g}^{-1} \mathrm{C}$, respectively for P-rich and P-poor Selenastrum cultures). Algae from all cultures, i.e. from all $\mathrm{P}$ treatments and from continuous or batch cultures, were diluted to standardized particulate $\mathrm{C}$ concentrations of about $4 \mathrm{mg} \mathrm{l}^{-1}$ before being fed to the Daphnia cultures.

\section{Daphnia cultures and experimental conditions}

The zooplankters $D$. magna were taken from a non-monoclonal laboratory culture. They have been grown on $S$. capricornutum for $>5$ years. For our experiments, all neonates produced by several mothers in $<24 \mathrm{~h}$ were isolated and fed with P-rich algae in saturating concentrations. All experiments were performed at $20^{\circ} \mathrm{C}$ and subdivided into two treatments: animals fed HQ- or LQSelenastrum cells.

Ingestion experiment

Fifteen beakers per treatment were filled with $25 \mathrm{ml} \mathrm{LQ}$ - or HQunlabelled algae at $4 \mathrm{mg} \mathrm{C}^{-1}$. This concentration is far above the incipient limiting level for D. magna (Lampert 1987). Five 6-dayold Daphnia were gently transferred to each beaker and acclimatized to food concentration and quality. After $1 \mathrm{~h}$, unlabelled algae were replaced by labelled algae with the corresponding qualities. After $0,1,3,6$ and $10 \mathrm{~min}$, three beakers per treatment were filtered and all Daphnia were carefully rinsed in cold medium before being transferred to scintillation vials for liquid scintillation counting. Five millilitres from each beaker was sampled for counting food radioactivity. The mass-specific amount of ingested $\mathrm{C}$ was calculated for each beaker by the following equation:

$I=\frac{A_{\mathrm{zoo}}}{S A N W} 100$

where $I=$ mass-specific amount of ingested C (\% of body $\mathrm{C}$ ), $A_{\text {zoo }}=$ activity in daphniids $(\mathrm{dpm}), S A=$ specific activity in algae (dpm $\left.\mu \mathrm{g}^{-1} \mathrm{C}\right), N=$ number of valid Daphnia in the beaker (individuals; ind), $W=$ mean body weight of a 6-day-old Daphnia 
$\left(\mu \mathrm{g} \mathrm{C}\right.$ ind $\left.^{-1}\right)$. The mass-specific ingestion rate of Daphnia was calculated for each treatment by a linear least-squares regression of mass-specific amounts of ingested $\mathrm{C}$ against feeding time on labelled algae.

\section{Assimilation experiment}

Four beakers per treatment were filled with $50 \mathrm{ml}$ LQ- or HQlabelled algae. Ten 6-day-old Daphnia were gently transferred to each beaker. After $1 \mathrm{~h}$ and $3 \mathrm{~h}$, two beakers per treatment were filtered and the animals were well rinsed, harvested in groups of two to six, and their radioactivity measured. Samples of water were taken at the start and at the end of each incubation for counting $\mathrm{C}$ and radioactivity in algae. The net $\mathrm{C}$ assimilation rate was calculated for each Daphnia activity count by:

net $\rho_{01}=\left(\frac{A_{x}}{S A N W} 100-D T\right) \frac{1}{t}$

where net $\rho_{01}=$ net $\mathrm{C}$ assimilation rate $\left(\%\right.$ of body $\left.\mathrm{C} \mathrm{h}^{-1}\right), A_{x}=$ activity in Daphnia after $x$ hours (dpm), $N=$ number of harvested Daphnia (ind), DT=estimated $\mathrm{C}$ mass in digestive tract (\% of body $\mathrm{C}$ ), $t=$ time $(1$ or $3 \mathrm{~h})$. The $y$-intercept of a linear least-squares regression of all values of $\frac{A_{X}}{S A N W} 100(\%$ of body C) versus time of incubation was considered as a good approximation of DT. As a part of the freshly assimilated $\mathrm{C}$ is already respired during the incubations, we need to correct the net assimilation values for ${ }^{14} \mathrm{C}$ losses before estimation of total assimilation rates (Lampert 1977). We used respiration rates calculated from the respiration experiment (see below) to estimate the losses. So, the total $\mathrm{C}$ assimilation rate is expressed as:

$\rho_{01}=\frac{T A_{x}}{t}$

where $\rho_{01}=$ total $\mathrm{C}$ assimilation rate $\left(\%\right.$ of body $\left.\mathrm{C} \mathrm{h}^{-1}\right), T A_{x}=$ total assimilated $\mathrm{C}$ after $x$ hours (\% of body $\mathrm{C}$ ), with:

$T A_{x}=\left(\frac{A_{x}}{S A N W} 100-D T_{c}\right)\left(1+L_{x}\right)$

where $D T_{c}=$ corrected $\mathrm{C}$ mass in digestive tract (\% of body C), $L_{x}=$ estimated relative losses by respiration of freshly assimilated ${ }^{14} \mathrm{C}$ after $x$ hours (no units), defined by Eq. 6 (see below). $D T_{c}$ was estimated by calculating the $y$-intercept of a linear least-squares regression of all values of $T A_{1}$ and $T A_{3}$ versus time. As knowledge of $D T_{c}$ is needed to calculate $T A_{x}$, and vice versa, both values were successively processed until stabilisation to the third decimal was reached.

\section{Respiration/excretion experiment}

One-day-old Daphnia were fed with ${ }^{14} \mathrm{C}$ labelled P-rich Selenastrum. This procedure made it possible to obtain a perfectly stable specific activity in animals after only 4 days (data not shown). Sixday-old labelled Daphnia were gently rinsed and incubated in groups of five in beakers filled with $10 \mathrm{ml} \mathrm{HQ}$ - or LQ-unlabelled algal suspensions. Five beakers were used per treatment. All incubations were made in the dark to avoid re-incorporation of ${ }^{14} \mathrm{CO}_{2}$ by algae. One Daphnia was collected with $2 \mathrm{ml}$ suspension from each beaker after 10, 20,30,60 and $120 \mathrm{~min}$. Daphnia radioactivity, total dissolved ${ }^{14} \mathrm{C}$ and $\mathrm{DO}^{14} \mathrm{C}$ were measured. We made use of the expected increases in both ${ }^{14} \mathrm{CO}_{2}$ and $\mathrm{DO}^{14} \mathrm{C}$ in the beakers so as to estimate, respectively, respiration and DOC excretion rates. ${ }^{14} \mathrm{CO}_{2}$ and $\mathrm{DO}^{14} \mathrm{C}$ data were first expressed in percentage of initial radioactivity in the Daphnia, and as animals were homogeneously labelled then in percentage of body $\mathrm{C}$.

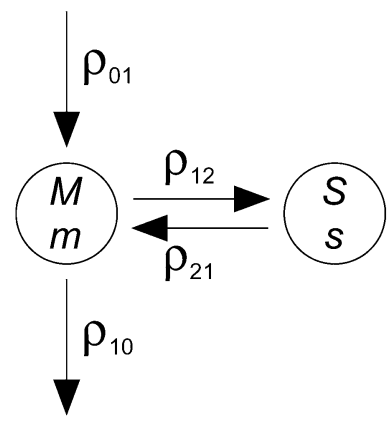

Fig. 1 Two-compartment model for tracer kinetics in Daphnia (modified from Lampert and Gabriel 1984). $\rho_{01} \mathrm{C}$ assimilation rate, $\rho_{12}$ transfer rate of $\mathrm{C}$ from metabolic to structural pool, $\rho_{21}$ transfer rate of $\mathrm{C}$ from structural to metabolic pool, $\rho_{10}$ respiration rate, $S$ relative size of structural pool, $s$ quantity of radioactive tracer in structural pool, $M$ relative size of metabolic pool, $m$ quantity of radioactive tracer in metabolic pool

\section{Analytical protocols}

\section{Radioactivity counting}

Animals were dissolved in $1 \mathrm{ml}$ of tissue solubilizer (Soluene-350, Packard), and were dried overnight at $60^{\circ} \mathrm{C}$. The water samples for counting specific activity in algae were filtered on GF/F Whatman filters. Filters were well rinsed with $0.1 \mathrm{~N} \mathrm{HCl}$ before counting. Radioactivity in filtered water was measured with or without addition of $25 \mu \mathrm{l} \mathrm{ml}^{-1}$ of $1 \mathrm{~N} \mathrm{HCl}$. The acidified water was gently shaken overnight before counting. The radioactivity in filtered water without acid was due to total dissolved ${ }^{14} \mathrm{C}$ while the radioactivity in acidified water resulted from $\mathrm{DO}^{14} \mathrm{C}$. The difference between both is a measure of ${ }^{14} \mathrm{CO}_{2}$. All scintillation vials were filled with $10 \mathrm{ml}$ of an environmentally friendly liquid scintillation cocktail (Ultima Gold, Packard) and were counted for radioactivity with a Packard scintillation counter. Quenching was corrected by a procedure using an internal standard.

\section{Elemental analyses}

Algal cells were collected on acid-washed and pre-ignited GF/F Whatman filters. Six-day-old Daphnia, reared in parallel to those used in experiments, were placed in tin capsules and dried $\left(60^{\circ} \mathrm{C}\right.$, 24 h). Particulate $C$ in both algae and Daphnia was analysed on a Carlo-Erba CHN 1106 elemental analyser. Total P was analysed by a modified procedure with peroxi-disulphate digestion followed by spectrophotometric determination of phosphate (Hessen et al. 2002).

\section{Tracer kinetic modelling}

The results of the respiration experiment, i.e. the increase in ${ }^{14} \mathrm{CO}_{2}$ in water or the corresponding decrease in Daphnia's body radioactivity due to respiration, were fitted with an adjustment of Lampert and Gabriel's (1984) model. The resulting respiration rates were used to estimate the relative losses of freshly assimilated labelled $\mathrm{C}$ in the assimilation experiment. The physiological model of Lampert and Gabriel (1984), presented in Fig. 1, is based on the assumption that the kinetics of the $\mathrm{C}$ tracer in small zooplankton is well described by a two-compartment model consisting of a small metabolic pool with a fast turnover rate and a large structural pool with a slow turnover rate (Conover and Francis 1973; Lampert 1975) 
Table 1 Presentation of symbols used in the two-compartment model for tracer kinetics in Daphnia

\begin{tabular}{lll}
\hline Symbols & Descriptions & Units \\
\hline$M$ & Relative size of metabolic pool & $\%$ of total body C \\
$S$ & Relative size of structural pool & $\%$ of total body C \\
$m$ & Quantity of radioactive tracer in metabolic pool & $\mathrm{dpm}$ \\
$s$ & Quantity of radioactive tracer in structural pool & $\mathrm{dpm}$ \\
$\rho_{01}$ & C assimilation rate & $\%$ of total body c $\mathrm{h}^{-1}$ \\
$\rho_{12}$ & Transfer rate of C from metabolic to structural pool & $\%$ of total body C $\mathrm{h}^{-1}$ \\
$\rho_{21}$ & Transfer rate of C from structural to metabolic pool & $\%$ of total body C $\mathrm{h}^{-1}$ \\
$\rho_{10}$ & Respiration rate & $\%$ of total body C $\mathrm{h}^{-1}$ \\
$t$ & Time & $\mathrm{h}$ \\
$m_{0}$ & Quantity of radioactive tracer in metabolic pool at start of & $\mathrm{dpm}$ \\
& respiration/excretion experiment & $\mathrm{dpm}$ \\
$s_{0}$ & Quantity of radioactive tracer in structural pool at start of & \\
$(m+s)_{0}$ & respiration/excretion experiment & Quantity of radioactive tracer in total body at start of \\
$W$ & respiration/excretion experiment & $\mathrm{dpm}$ \\
$S A$ & Body weight of aDaphnia & $\mu \mathrm{g} \mathrm{C}$ \\
& Specific activity in algae & $\mathrm{dpm} \mu \mathrm{g}^{-1} \mathrm{C}$ \\
\hline
\end{tabular}

\section{Modelling of kinetics in the respiration experiment}

We first used this model to develop equations for calculating the variations of radioactivity over time in homogeneously labelled Daphnia feeding on unlabelled algae (as was the case in the respiration experiment). The entire demonstration of the model is presented in Appendix A. All symbols are explained in Table 1.

The kinetic of total Daphnia radioactivity, $m+s$, as a function of time is described by:

$$
\begin{gathered}
m+s=a_{1} \exp \left(\lambda_{1} t\right)\left(1+\frac{\rho_{12} / M}{\lambda_{1}+\rho_{21} / S}\right) \\
+a_{2} \exp \left(\lambda_{2} t\right)\left(1+\frac{\rho_{12} / M}{\lambda_{2}+\rho_{21} / S}\right)
\end{gathered}
$$

with:

$$
\begin{array}{lll}
a_{1} & =m_{0}-a_{2} & \lambda_{1} \text { and } \lambda_{2} \text { are as } \\
a_{2} & =\left[\frac{\lambda_{1}+\rho_{21} / S}{\rho_{12} / M} s_{0}-m_{0}\right] \frac{\lambda_{2}+\rho_{21} / s}{\lambda_{1}-\lambda_{2}} & \\
\lambda_{1} & =-\frac{1}{2}\left(\rho_{12} / M+\rho_{21} / S+\rho_{10} / M\right)+\frac{1}{2} \sqrt{\left(\rho_{12} / M+\rho_{21} / S+\rho_{10} / M\right)^{2}-4 \rho_{10} \rho_{21} / M S} \\
\lambda_{2} & =-\frac{1}{2}\left(\rho_{12} / M+\rho_{21} / S+\rho_{10} / M\right)-\frac{1}{2} \sqrt{\left(\rho_{12} / M+\rho_{21} / S+\rho_{10} / M\right)^{2}-4 \rho_{10} \rho_{21} / M S}
\end{array}
$$

Thus, Eq. 4 is based on seven parameters: $M, S, m_{0}, s_{0}, \rho_{10}, \rho_{21}$ and $\rho_{12} . M$ and $S$ are assumed to be absolutely invariant, and well estimated by Lampert and Gabriel (1984), equalling 1.6 and 98.4, respectively. $m_{0}$ and $s_{0}$ are measured at the start of the experiment:

$$
m_{0}=(m+s)_{0} M \quad \text { and } s_{0}=(m+s)_{0} S
$$

The three remaining parameters, $\rho_{10}, \rho_{21}$ and $\rho_{12}$, are connected by (Lampert and Gabriel 1984):

$\rho_{12}=\frac{S}{M+S}\left(\rho_{01}-\rho_{10}\right)+\rho_{21}$

After substitution of $\rho_{12}$ in Eq. 4 by Eq. 5, and as the C assimilation rate, $\rho_{01}$, is estimated from the assimilation experiment, we are left in Eq. $4(m+s)$ with two unknowns, $\rho_{10}$ and $\rho_{21}$, that were estimated by fitting experimental data from the respiration experiment with Eq. 4. The experimentally observed values for $(m+s)$ were obtained by subtracting respired ${ }^{14} \mathrm{CO}_{2}$ (in dpm) from $(m+s)_{0}$.

\section{Modelling of kinetics in the assimilation experiment}

The same model was again used to develop equations for calculating the variations of radioactivity over time in each pool of unlabelled Daphnia feeding on labelled algae (as was the case in the assimilation experiment). The entire demonstration of the model is presented in
Appendix B. The model was used to estimate the relative losses by respiration of freshly assimilated ${ }^{14} \mathrm{C}$ after $x$ hours, $L_{x}$ :

$L_{x}=\frac{\int_{0}^{x} \frac{\rho_{10}}{M} m_{t} d t}{m_{x}+s_{x}}$

with:

$$
\begin{aligned}
m_{t} & =a_{1} \exp \left(\lambda_{1} t\right)+a_{2} \exp \left(\lambda_{2} t\right)+\frac{\rho_{01}}{\rho_{10} 100} W M S A \\
s_{t} & =\frac{\rho_{12} a_{1} / M}{\lambda_{1}+\rho_{21} / s} \exp \left(\lambda_{1} t\right)+\frac{\rho_{12} a_{2} / M}{\lambda_{2}+\rho_{21} / s} \exp \left(\lambda_{2} t\right)+\frac{\rho_{12} \rho_{01}}{\rho_{10} \rho_{21} 100} W S S A \\
a_{1} & =-\left(a_{2}+\frac{\rho_{01}}{\rho_{10} 100} W M S A\right) \\
a_{2} & =\frac{\rho_{01}}{\rho_{10} 100} W M S A\left(1-\frac{\lambda_{1}+\rho_{21} / s}{\rho_{21} / S}\right) \frac{\lambda_{2}+\rho_{21} / s}{\lambda_{1}-\lambda_{2}}
\end{aligned}
$$

$\lambda_{1}$ and $\lambda_{2}$ are as defined above.

\section{Repeated estimation of the rates}

In Eq. 6 the respiration rate $\rho_{10}$ is used for evaluating respiratory losses $L_{x}$ (needed for a correct estimation of the total $\mathrm{C}$ assimilation rate $\rho_{01}$, see Eqs. 2 and 3) while at the same time the determination of $\rho_{01}$ is required for the fitting of Eq. 4 and then estimation of $\rho_{10}$. Therefore, an iterative process was computed. Firstly, the data set of the assimilation experiment was used for estimating $\rho_{01}$ by Eqs. 2 and 3 but without correction for respired C. Secondly, the data set of the respiration experiment and the value found for $\rho_{01}$ were used for estimating $\rho_{10}$ and $\rho_{21}$ by Eq. 4 , and $\rho_{12}$ by Eq. 5 . The values found were then inserted in Eq. 6 to obtain the relative quantity of freshly assimilated $\mathrm{C}, L_{x}$, that had already been respired. This was then re-used for estimating $\rho_{01}$, and so forth. The iterative process was pursued until stabilisation at the third decimal of all values.

\section{DOC excretion modelling}

In order to estimate DOC excretion rates from labelled Daphnia feeding on unlabelled algae, we have applied a model describing the kinetics of $\mathrm{DO}^{14} \mathrm{C}$ accumulation in water during incubations. As there was no time lag during transfer from labelling culture to 
unlabelled algae except for $30 \mathrm{~s}$ of washing in COMBO medium, the digestive tracts of animals were most probably initially filled with labelled algae. As a consequence, $\mathrm{DO}^{14} \mathrm{C}$ entering the water during incubations could be from two sources: release in a dissolved form of organic ${ }^{14} \mathrm{C}$ from digestive tracts or faeces, and actual secretion of metabolised organic C-rich compounds from labelled body tissues. As gut passage time of D. magna is about $10 \mathrm{~min}$, and knowing the extremely rapid diffusion of dissolved compounds from zooplankton faeces $(90 \%$ in $<1 \mathrm{~s}$ for this kind of faeces; Jumars et al. 1989), we used the accumulation of $\mathrm{DO}^{14} \mathrm{C}$ only during the 20-min to 2-h interval as the basis for our model, assuming that this was primarily accounted for by metabolised DOC. Accumulation of $\mathrm{DO}^{14} \mathrm{C}$ in water over time was matched by a saturation curve with a positive non-zero intercept on the $y$-axis. This intercept, $F$, corresponds to the quantity of unassimilated organic ${ }^{14} \mathrm{C}$ leaked from digestive tracts or faeces. The curvature parameter, $\rho_{\mathrm{DOC}}$, corresponds to the actual excretion rate of DOC from body tissues, and the saturation level, $D$, must be equal to the size of the kinetically homogeneous metabolic pool from which organic C is excreted. As Daphnia labelling was identical for all animals irrespective of the treatment they underwent during the excretion experiment, we can postulate that $F$ was similar between treatments. Data from both treatments were thus used simultaneously for estimating $F$, whilst excretion rates and $D$ were assumed to be treatment specific. Data were fitted by:

$$
\begin{aligned}
\text { DOCe }= & F+\alpha D_{\mathrm{HQ}}\left(1-e^{-\frac{\rho_{\mathrm{DOC}-\mathrm{HQ}}}{D_{\mathrm{HQ}}} t}\right) \\
& +(1-\alpha) D_{\mathrm{LQ}}\left(1-e^{-\frac{\rho_{\mathrm{DOC}-\mathrm{LQ}}}{D_{\mathrm{LQ}}} t}\right)
\end{aligned}
$$

where $D O C e=$ total excreted $\mathrm{DO}^{14} \mathrm{C}$ after time $t$ for both treatments (\% of total body $\mathrm{C}$ ), $F$ =quantity of unassimilated organic ${ }^{14} \mathrm{C}$ leaked from digestive tracts or faeces (\% of total body $\mathrm{C}$ ), $\alpha=$ dummy variable, coded 0 for LQ data and 1 for HQ data (no units), $D_{\mathrm{HQ}}=$ size of metabolic pool source of organic $\mathrm{C}$ excretion in HQ treatment ( $\%$ of total body C), $D_{\mathrm{LQ}}=$ size of metabolic pool source of organic $\mathrm{C}$ excretion in LQ treatment (\% of total body $\mathrm{C}$ ), $\rho_{\mathrm{DOC}-\mathrm{HQ}}=\mathrm{DOC}$ excretion rate in HQ treatment $\left(\%\right.$ of total body $\mathrm{C} \mathrm{h}^{-}$ $\left.{ }^{1}\right), \rho_{\text {DOC-LQ }}=$ DOC excretion rate in LQ treatment ( $\%$ of total body $\mathrm{C}$ $\left.\mathrm{h}^{-1}\right), t$ in hours. We did not apply a multiple-compartment model for DOC kinetics, as we did for $\mathrm{CO}_{2}$ kinetics, because the published background literature is still insufficient. Thus, this one-compartment model does not take into account any transfer during incubations of organic labelled compounds from another pool to $D$.

\section{Results}

\section{Ingestion}

The incorporation of labelled algae was linear for the three first samples (up to $6 \mathrm{~min}$ ) (Fig. 2). Judging from the increased variability of the 10-min sample, and also from the reduced rate of increase, some animals had a gut passage time $<10 \mathrm{~min}$, and thus the 10-min set was excluded from the regression on ingestion values. Ingestion rates did not differ between treatments (two-tailed test for parallelism, $P>0.60$; Fig. 3A).

\section{Assimilation}

The net assimilation rates were calculated by Eq. 1 (Fig. 4A). These rates differed significantly between treatments (two-tailed $t$-test, $P<0.001$ ). We used respira-

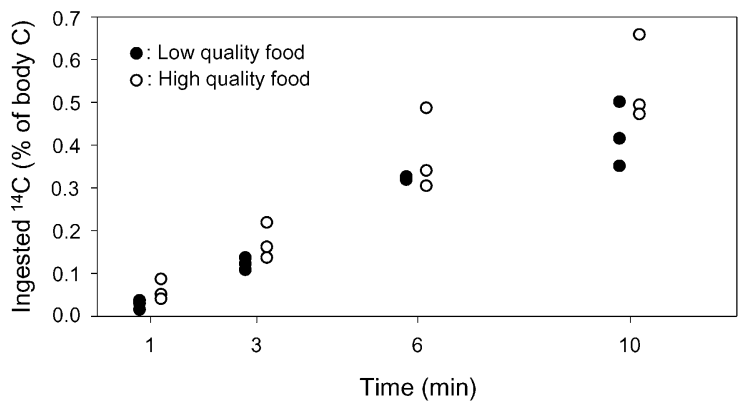

Fig. 2 Incorporation of ${ }^{14} \mathrm{C}$ by unlabelled Daphnia feeding on labelled algae. Each value represents one beaker with five Daphnia. Calculated ingestion rates are presented in Fig. 3A

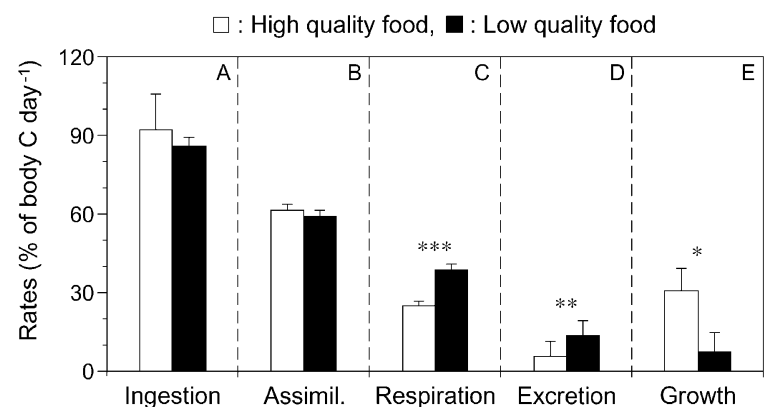

Fig. 3A-E Synthesis of estimated rates in Daphnia. A Ingestion rate, $\mathbf{B}$ total assimilation (Assimil.) rate, $\mathbf{C}$ respiration rate, $\mathbf{D}$ dissolved organic C (DOC) excretion rate, $\mathbf{E}$ growth rate. Vertical bars represent $1 \mathrm{SE}$. Respiration and DOC excretion rates were statistically higher in the low quality treatment, while growth rate was lower (one-tailed $t$-test, $* P<0.05, * * P<0.025$, $* * * P<0.001$ )

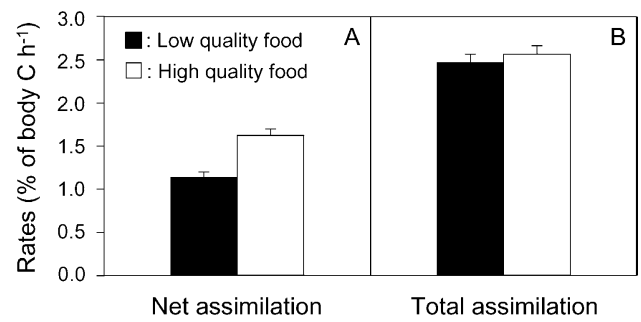

Fig. 4A, B Assimilation rates of ${ }^{14} \mathrm{C}$ by unlabelled Daphnia feeding on labelled algae. Vertical bars represent 1 SE. A Net assimilation rates are calculated without correction of incorporation values for ${ }^{14} \mathrm{C}$ losses by respiration. They are highly significantly different between treatments (two-tailed $t$-test, $P<0.001$ ). B Total assimilation rates are calculated after correction for respiratory losses. They are not significantly different between treatments $(P>0.50)$

tion rates calculated from the respiration experiment (see below) to estimate losses by respiration of freshly assimilated ${ }^{14} \mathrm{C}$ during assimilation incubations. Equation 6 yielded respiratory losses of ${ }^{14} \mathrm{C}$ equal to $19.6 \%$ and $29.1 \%$ of net assimilated ${ }^{14} \mathrm{C}$ after respectively 1 and $3 \mathrm{~h}$ of incubation for the HQ treatment, and 41.7 and $78.9 \%$ for the LQ treatment. After correcting net assimilated ${ }^{14} \mathrm{C}$ data for respiration losses (see Eqs. 2 and 3), the difference in total $\mathrm{C}$ assimilation rate between $\mathrm{HQ}$ and 


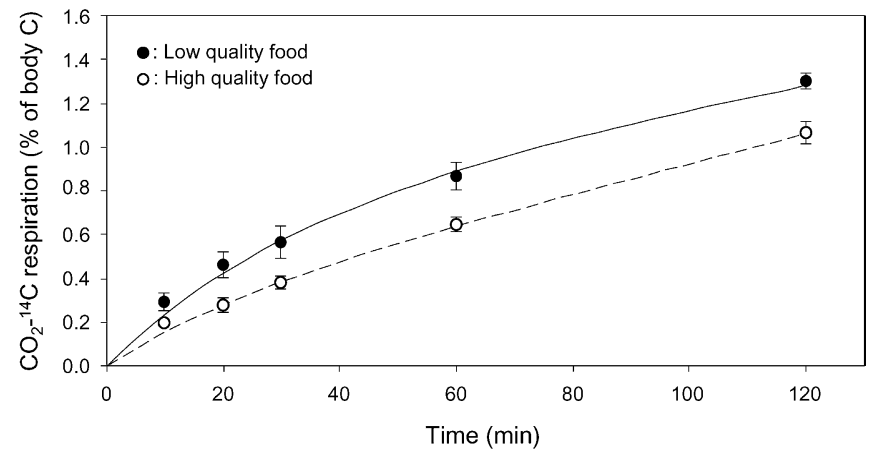

Fig. 5 Respiration of ${ }^{14} \mathrm{CO}_{2}$ by labelled Daphnia feeding on unlabelled algae. Vertical bars represent \pm 1 SE. Lines are adjusted by modelling, not by regressions. Calculated respiration rates are presented in Fig. 3C

Table 2 Results of the assimilation and respiration/excretion experiments of Daphnia magna feeding on both Selenastrum capricornutum cultures of varying phosphorus quality. Algal C:P ratio was equal to 80 and 400, respectively in high quality (HQ) and low quality (LQ) treatments. $\mathrm{C}$ assimilation rates $\left(\rho_{01}\right)$, respiration rates $\left(\rho_{10}\right)$, transfer rates of $\mathrm{C}$ from structural to metabolic pools $\left(\rho_{21}\right)$, transfer rates of $\mathrm{C}$ from metabolic to structural pool $\left(\rho_{12}\right)$ and DOC excretion rates $\left(\rho_{\text {DOC }}\right)$ are in $\%$ of body $C$ individual ${ }^{-1} \mathrm{~h}^{-1}$. The quantity of unassimilated organic ${ }^{14} \mathrm{C}$ leaked from digestive tracts or faeces $(F)$ and sizes of metabolic pool source of organic $\mathrm{C}$ excretion $\left(D_{\mathrm{HQ}}\right.$ and $\left.D_{\mathrm{LQ}}\right)$ are in $\%$ of body $\mathrm{C}$ individual ${ }^{-1}$. Data are means \pm 1 SE. Values are obtained by either direct measurement $(\mathrm{M})$, indirect calculation (C) or fitting with experimental observations $(\mathrm{F})$

\begin{tabular}{llll}
\hline & HQ food & LQ food & \\
\hline Respiration & & & \\
$\rho_{01}$ & $2.56 \pm 0.10$ & $2.47 \pm 0.10$ & $\mathrm{M}$ \\
$\rho_{10}$ & $1.05 \pm 0.07$ & $1.61 \pm 0.10$ & $\mathrm{~F}$ \\
$\rho_{21}$ & $1.58 \pm 0.41$ & $0.56 \pm 0.23$ & $\mathrm{~F}$ \\
$\rho_{12}$ & $3.06 \pm 0.42$ & $1.41 \pm 0.26$ & $\mathrm{C}$ \\
Excretion & & & \\
$F$ & $0.38 \pm 0.09$ & & $\mathrm{~F}$ \\
$\rho_{\mathrm{DOC}}$ & $0.24 \pm 0.23$ & $0.56 \pm 0.23$ & $\mathrm{~F}$ \\
$D_{\mathrm{HQ}}$ and $D_{\mathrm{LQ}}$ & $6.94 \pm 165$ & $10.0 \pm 65.1$ & $\mathrm{~F}$ \\
\hline
\end{tabular}

LQ treatments became insignificant $(P>0.50$, Fig. $4 \mathrm{~B})$. Thus, gross $\mathrm{C}$ assimilation efficiencies $(69.3 \%$ and $68.0 \%$, respectively, for $\mathrm{LQ}$ and $\mathrm{HQ}$ treatments) were similar between treatments.

\section{Respiration}

Higher accumulations of ${ }^{14} \mathrm{CO}_{2}$ were found in the beakers where Daphnia were fed with LQ food compared with the HQ treatment (Fig. 5). This suggests a higher respiration rate for Daphnia fed with low-P algae. Our data gave close fits with the two-compartment model $\left(r^{2}=0.90\right.$ and 0.95 , respectively, for LQ and HQ treatments). Fitting data allowed us to estimate $\rho_{10}$ and $\rho_{21}$ for both treatments, and then $\rho_{12}$ was calculated by Eq. 5 (Table 2 ). The estimated respiration rate $\left(\rho_{10}\right)$ for the LQ treatment

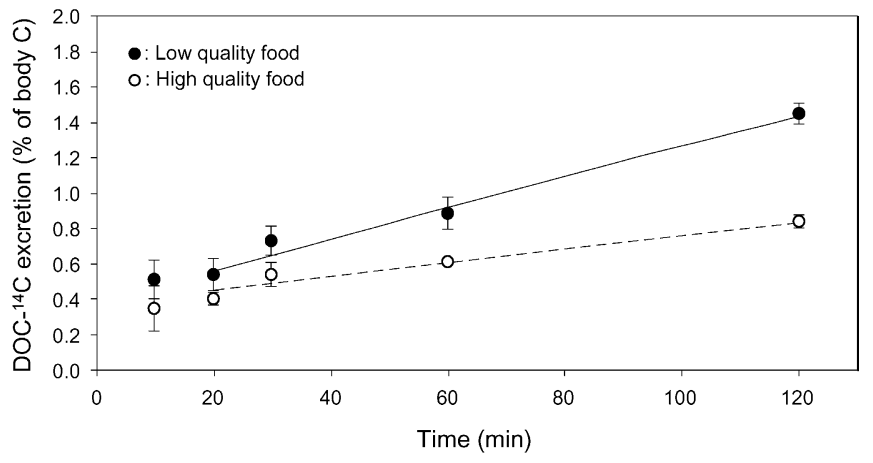

Fig. 6 Excretion of $\mathrm{DO}^{14} \mathrm{C}$ by labelled Daphnia feeding on unlabelled algae. Vertical bars represent \pm 1 SE. Lines are adjusted by modelling, not by regressions. Values at time $=10 \mathrm{~min}$ are plotted, but were not used for modelling. Calculated DOC excretion rates are presented in Fig. 3D

was higher than the one in the HQ treatment (one-tailed $t$ test, $P<0.001$, Fig. 3C). However, the transfer rate between metabolic and structural pools $\left(\rho_{12}\right)$ and the inverse flux $\left(\rho_{21}\right)$ were both much lower in the LQ than in the HQ treatment $\left(P<0.001\right.$ for $\rho_{12}, P<0.025$ for $\left.\rho_{21}\right)$. It is worth mentioning that, after $10 \mathrm{~min}$ of incubation, the amount of ${ }^{14} \mathrm{CO}_{2}$ released by Daphnia was already higher in the LQ treatment than in the HQ treatment $(P<0.025)$.

\section{DOC excretion}

As for release of ${ }^{14} \mathrm{CO}_{2}$, accumulations of $\mathrm{DO}^{14} \mathrm{C}$ in beakers differed significantly (two-tailed $t$-test, e.g. $P<0.05$ for 1 -h data and $P<0.001$ for 3 -h data) indicating a higher DOC excretion rate for Daphnia feeding on Pdeficient algae (Fig. 6). The $y$-variance of these data was well explained by the model of Eq. $7\left(r^{2}=0.78\right.$ and 0.73 , respectively, for LQ and HQ treatments). Results of modelling are presented in Table 2. The estimated excretion rate of DOC was higher in the LQ treatment than in the HQ treatment (one-tailed $t$-test, $P<0.025$, Fig. 3D).

\section{Discussion}

The offered food "quality" in our experiments represented a C:P ratio (by atoms) of 80 and 400 . Food concentrations were kept above the incipient limiting level. Using Sterner's (1997) model, we calculated the food C:P threshold for the onset of $\mathrm{P}$ limitation in the consumer. When using our ingestion, assimilation and respiration data, an atomic C:P ratio in D. magna of 80 (Vrede et al. 1999) and a P assimilation efficiency of 0.8 (DeMott et al. 1998), we obtained food C:P thresholds between 120 and 160. Thus, while a $C: P$ of 400 is well below the applied maximum of ca. 1,500 that may be observed in $S$. capricornutum under severe $\mathrm{P}$ deficiency (Hessen et al. 2002), our LQ treatment should nevertheless represent 
food quality causing $\mathrm{P}$ limitation and thus $\mathrm{C}$ in excess. Inversely, the food C:P ratio in the HQ treatment (80) was well below these limits and thus growth of Daphnia in this case was not limited by P. All experiments were made with animals grown on P-saturated food in order to obtain equally fit animals, and thus to highlight the immediate effects of dietary $\mathrm{P}$ depletion on digestion and respiration traits. Resource depression is a common seasonal process in nature (see e.g. Sterner 1998).

\section{Ingestion and assimilation}

Filtration and $\mathrm{C}$ ingestion rates measured in our shortterm incubations $(<10 \mathrm{~min})$ were not affected by food quality. These observations agree with results from shortterm experiments with the rotifer Brachionus rubens (Rothhaupt 1995) and D. magna (DeMott et al. 1998). Yet some studies have shown apparently reduced algal clearance rates in long-term (20 h-2 day) incubations of daphniids feeding on P-limited chlorophytes (Van Donk and Hessen 1993; Sterner et al. 1993; Sterner and Smith 1993). So far these results do not really offer the possibility to draw conclusions about the regulation of ingestion rates because, as already pointed out by Sterner and Hessen (1994), these data were influenced by the gut passage of intact algal cells as observed for extremely Plimited algae $(\mathrm{C}: \mathrm{P}>1,000)$ in batch culture (Van Donk and Hessen 1993). Experiments using semicontinuous cultures or chemostats have been unable to verify this effect (DeMott et al. 1998; Hessen et al. 2002).

In our tested range of algal C:P ratios (80-400), we failed to observe any difference in total $\mathrm{C}$ assimilation rate and $\mathrm{C}$ assimilation efficiency between treatments in D. magna, in contrast with DeMott et al. (1998). In experiments with Scenedesmus as food they observed a constant decrease in $\mathrm{C}$ assimilation efficiency (from 0.92 to 0.50 ) when food $\mathrm{C}: \mathrm{P}$ increased from 80 to 475 (see their Fig. 7). This could in part be attributed to differing algal species. Moreover our estimated assimilation rates were based on ingestion of labelled food during a 3-h period corrected for $\mathrm{C}$ losses due to respiration during incubations. By contrast, the rates measured by DeMott et al. (1998) were not corrected for respiratory losses of C from the metabolic pool during their 40-min incubations. As we observed an increased respiration rate in the LQ treatment, the reduced accumulation (net assimilation) of labelled $\mathrm{C}$ in the LQ treatment relative to the HQ one would also be due to an immediate loss of label from the metabolic pool. If the increased respiration rate is not corrected for, this will cause underestimation of assimilation rates when food C:P increases. This alone could explain the differences in assimilation rates that DeMott et al. (1998) observed between food of contrasting C:P ratios. Also the rapid decline of the apparent assimilation rates observed in their experiment after only $30 \mathrm{~min}$ of feeding with P-deficient algae could be explained by rapid increases in respiration rate, as observed in our experiment after only $10 \mathrm{~min}$.

\section{Respiration}

The two-compartment model (Lampert and Gabriel 1984) described satisfactorily the kinetics of the radioactive $\mathrm{C}$ tracer in Daphnia (see Fig. 5). The $\mathrm{CO}_{2}$ excretion rate we measured for P-saturated algae (HQ treatment) is very similar to that observed by Lampert and Bohrer (1984) in the same species at high algal biomass, i.e. $25.3 \%$ and $23.5 \%$, respectively, of total body $\mathrm{C}$ day $^{-1}$. A deficiency of $\mathrm{P}$ in the diet (LQ treatment) led to a higher respiration rate $\left(38.7 \%\right.$ of total body $\mathrm{C}$ day $^{-1}$ ) and to an important decrease in transfer rates between metabolic and structural pools. Thus, when food becomes P-limited, more C is lost through respiration, and less $\mathrm{C}$ is allocated to the body structure. This important decrease in transfer rate between metabolic and structural pools could explain the reported lower growth rates when herbivores feed on mineral-deficient food (see e.g. Sterner et al. 1993; Hessen et al. 2002). We do not know whether this represents a transient situation, however. We transferred animals from labelled HQ food to unlabelled LQ food, and thus a full metabolic adjustment to LQ food may not have occurred. It is not clear how this increased respiration (and thus extra gain of energy and ATP) may affect the animals in the long run.

Plath and Boersma (2001) observed highly increased appendage beat rates of $D$. magna feeding on P-deficient algae in comparison with when feeding on algae richer in P. The authors suggested that the predicted resulting increase in filtration rate, an energy (thus C) costly process, offers a mechanistic explanation of the observed relative homeostasis of the animal. Our data confirm that the increase in the respiration rate is a process used by this species when facing a dietary nutrient deficiency, but they offer no identification of the energy expending process. We measured the filtration rate only during the first 10 min after the transfer to the LQ food and observed no difference with the one for HQ algae, whereas the respiration rates were estimated over a period of $2 \mathrm{~h}$. Of course, we do not know how the filtration rate may have evolved after the first $10 \mathrm{~min}$. But the fact that already after 10 min the respiration rate in the treatment with $\mathrm{LQ}$ algae was higher than in the one with HQ food suggests that filtration is certainly not the only energy expending process which increases in the case of dietary nutrient deficiency.

Our observation of increased respiration rate in a crustacean zooplankter when feeding on mineral-deficient algae supports data on heterotrophic bacteria. Both theoretical and experimental studies have demonstrated higher individual respiration rates and lower gross growth efficiencies in marine bacteria when substrate $\mathrm{C}: \mathrm{N}$ ratios increase (Harder and Dijkhuizen 1983; Billen 1984; Lancelot and Billen 1985; Goldman et al. 1987; Hopkinson et al. 1989). Similar trends were more recently observed for freshwater bacteria with increasing substrate $\mathrm{C}: \mathrm{P}$ and C:N ratios (Cimbleris and Kalff 1998; Biddanda et al. 2001). Higher seston $C: N$ or $C: P$ ratios indicate lower substrate availability for bacteria, which generates 
heavier costs of biosyntheses and increases catabolism of C compounds (Goldman et al. 1987; Cimbleris and Kalff 1998). These effects could thus be a general property for heterotrophs facing an unbalanced diet with high C:P or $\mathrm{C}: \mathrm{N}$. Note that the same principle may hold for photorespiration observed in green algae and $\mathrm{C}-3$ higher plants when improving high light levels. Although resorting to a completely different biochemical pathway, this extracellular release of $\mathrm{CO}_{2}$ can also be described as a process preserved through evolution allowing organisms to maintain cellular homeostasis in case of high energy input (see e.g. Salisbury and Ross 1992; Reynolds 1997).

\section{DOC excretion}

To our knowledge, this is the first time that excretion rates of DOC directly released from body tissues have been estimated in crustacean zooplankton. In the literature, impacts of grazing on DOC stocks and bacterial uptake have often been mentioned (e.g. Hygum et al. 1997). Due to methodological constraints, however, the mechanisms of DOC release have rarely been investigated (Park et al. 1997, Strom et al. 1997). In experiments where animals are fed with labelled algae, the release of DOC from different sources (sloppy feeding, egestion of unassimilated compounds and release from faeces) has rarely been considered, and direct DOC excretion is rarely taken into account.

Nevertheless we can try to compare the DOC excretion rates we estimated with those of three previously published studies: Lampert (1978) and Olsen et al. (1986) who both estimated DOC release by using radiotracers, and Strom et al. (1997) who simply measured the quantity of released DOC with or without grazers. We indirectly estimated Daphnia pulex DOC excretion rates in Lampert's experiments (Lampert 1978) by calculating the mean difference between his second (long-term, $3 \mathrm{~h}$ ) and his first (short-term, $15 \mathrm{~min}$ ) series of incubations (see his Table 1). This difference is an indirect measurement of the fraction of the ingested food released as DOC by any process but that of sloppy feeding. It was found to be equal to $4.7 \%$ of ingested C. Olsen et al. (1986) found $\mathrm{DO}^{14} \mathrm{C}$ release rates in the same order of magnitude (5\% of ingested C) for D. magna feeding on unlabelled Scenedesmus acutus after $1 \mathrm{~h}$ of labelling. In our experiment, we estimated a very similar DOC excretion rate of $6.1 \%$ of ingested $\mathrm{C}$ when animals were fed on P-saturated algae (HQ treatment). We observed that Daphnia, when shifted to a diet of Pdeficient algae, increased its DOC excretion rate to $15.6 \%$ of ingested C (LQ treatment). This rather high rate is still within the range of previous studies. Strom et al. (1997) estimated Calanus pacificus DOC production due to direct excretion to be between 16 and $21 \%$ of ingested C. As for the respiration estimates above, it should also be remarked upon here that the high rate of DOC excretion observed in the LQ treatment could partly be a transient situation since animals were transferred from HQ food to
LQ food. Hence while the general difference between HQ and LQ treatments stands, the absolute rate in the LQ treatment should be treated with some caution.

The DOC excretion rate in D. magna was more than twice as high when it fed on LQ algae (high C:P ratio) than on P-rich algae. Thus, to regulate excess $\mathrm{C}$ in their diet, daphniids could greatly enhance the metabolization of stored or structural compounds assimilated before changes in the quality of food took place. It means that daphniids, depending on food quality, could be able to regulate their disposal of C-rich compounds through DOC excretion. Biochemical description of these excreted organic compounds as a function of food C:P ratio would be of great interest in the understanding of zooplankton grazing impact on bacterial productivity (Rosenstock and Simon 2001; Richardot et al. 2001).

Impact on growth rate and $\mathrm{C}$ flux

We calculated growth rate for both treatments as the difference between assimilation rate and loss rates (respiration and DOC excretion) (Fig. 3E). The observed differences in loss rates between treatments resulted in a fourfold reduction of Daphnia growth rate when feeding on LQ food (one-tailed $t$-test, $P<0.05$ ). Although this reduction supports the general finding that growth of Daphnia may be limited by low P in the food, the reduction of growth is more severe than reported in previous studies (e.g. Sterner 1993, Urabe et al. 1997, DeMott et al. 1998, Hessen et al. 2002). Growth rates cited in the literature are often obtained after long-term (at least a few days) incubations of animals, whereas in our study we calculated instantaneous rates obtained without prior acclimatization to food quality (with the exception of $1 \mathrm{~h}$ acclimatization for assimilation rates). Although different algae could yield different growth rates both under high and low C:P, it may also emphasize the phenotypic capacity of Daphnia to gradually implement other physiological mechanisms, like increased assimilation efficiency for $\mathrm{P}$ relative to $\mathrm{C}$, in order to tackle dietary deficiency.

In conclusion, our data suggest that increased respiration and increased release of C-rich compounds may be important mechanisms for metazoans to regulate excess $\mathrm{C}$ in food in order to maintain a rather rigid (though not totally homeostatic) control of elemental composition. In systems with temporal algal limitation by $\mathrm{P}$, the expected increases in respiration and DOC excretion by herbivorous zooplankton could probably increase $\mathrm{CO}_{2}$ output to the atmosphere, decrease zooplanktivorous fish production and favour the planktonic microbial loop. Since mineral limitation also seems to be a general phenomenon in marine and particularly terrestrial ecosystems (Elser and Hassett 1994; Elser et al. 2000), these regulation processes may potentially have great impacts on fluxes of C both on ecosystem and global scales. 
Acknowledgements The authors would like to thank Prof. J. Henrard for his help in resolving the equation systems. We are grateful to S. Diehl for his helpful suggestions and improvements to an earlier draft of the manuscript. F. D. was supported by an exchange Linkecol grant provided by the European Science Foundation, which funded travel and accommodation for the duration of the experiments at the University of Oslo.

\section{Appendix A}

Demonstration of equations modelling evolution over time of total radioactivity in homogeneously labelled Daphnia feeding on unlabelled algae

The kinetics of the tracer in each pool is described by a system of two differential equations (see Table 1 for explanation of symbols):

$\left\{\begin{array}{l}\frac{d(m)}{d t}=\frac{\rho_{21}}{S} s-\frac{\rho_{12}}{M} m-\frac{\rho_{10}}{M} m \\ \frac{d(s)}{d t}=\frac{\rho_{12}}{M} m-\frac{\rho_{21}}{S} s\end{array}\right.$

We know that this kind of differential system has a solution of the type:

$\left\{\begin{array}{l}m=a \exp \left(\lambda_{j} t\right) \\ s=b \exp \left(\lambda_{j} t\right)\end{array}\right.$

In using this type of solution in the system, we found:

$\left\{\begin{array}{l}\lambda_{j} a \exp \left(\lambda_{j} t\right)=\left[-\left(\frac{\rho_{12}+\rho_{10}}{M}\right) a+\frac{\rho_{21}}{S} b\right] \exp \left(\lambda_{j} t\right) \\ \lambda_{j} b \exp \left(\lambda_{j} t\right)=\left(\frac{\rho_{12}}{M} a-\frac{\rho_{21}}{S} b\right) \exp \left(\lambda_{j} t\right)\end{array}\right.$

In simplifying this by $\exp \left(\lambda_{j} t\right)$, we found algebraic equations for $a$ and $b$ :

$\left\{\begin{array}{l}-\left(\lambda_{j}+\frac{\rho_{12}+\rho_{10}}{M}\right) a+\frac{\rho_{21}}{S} b=0 \\ \frac{\rho_{12}}{M} a-\left(\lambda_{j}+\frac{\rho_{21}}{S}\right) b=0\end{array}\right.$

This system will give a non-trivial solution only if the next determinant

$$
\begin{aligned}
& \left|\begin{array}{cc}
-\left(\lambda_{j}+\frac{\rho_{12}+\rho_{10}}{M}\right) & \frac{\rho_{21}}{S} \\
\frac{\rho_{12}}{M} & -\left(\lambda_{j}+\frac{\rho_{21}}{S}\right)
\end{array}\right| \\
& =\lambda_{j}^{2}+\left(\rho_{12} / M+\rho_{21} / S+\rho_{10} / M\right) \lambda_{j}+\rho_{10} \rho_{21} / M S=0
\end{aligned}
$$

is equal to 0 . This gives a quadratic equation in $\lambda_{j}$ with two solutions:

$$
\begin{aligned}
\lambda_{1}= & -\frac{1}{2}\left(\rho_{12} / M+\rho_{21} / S+\rho_{10} / M\right) \\
& +\frac{1}{2} \sqrt{\left(\rho_{12} / M+\rho_{21} / S+\rho_{10} / M\right)^{2}-4 \rho_{10} \rho_{21} / M S}
\end{aligned}
$$

and

$$
\begin{aligned}
\lambda_{2}= & -\frac{1}{2}\left(\rho_{12} / M+\rho_{21} / S+\rho_{10} / M\right) \\
& -\frac{1}{2} \sqrt{\left(\rho_{12} / M+\rho_{21} / S+\rho_{10} / M\right)^{2}-4 \rho_{10} \rho_{21} / M S}
\end{aligned}
$$

We then rewrite the equations in system 8 (Eq. 8) under their decomposed form: $\left\{\begin{array}{l}m=a_{1} \exp \left(\lambda_{1} t\right)+a_{2} \exp \left(\lambda_{2} t\right) \\ s=b_{1} \exp \left(\lambda_{1} t\right)+b_{2} \exp \left(\lambda_{2} t\right)\end{array}\right.$

We then rewrite the second equation in system 9 (Eq. 9) with decomposition of $\lambda_{j}$ into $\lambda_{1}$ and $\lambda_{2}$ :

$\frac{\rho_{12}}{M} a_{1}-\left(\lambda_{1}+\frac{\rho_{21}}{S}\right) b_{1}=0 \Leftrightarrow b_{1}=\frac{\rho_{12} a_{1} / M}{\lambda_{1}+\rho_{21} / S}$

and

$\frac{\rho_{12}}{M} a_{2}-\left(\lambda_{2}+\frac{\rho_{21}}{S}\right) b_{2}=0 \Leftrightarrow b_{2}=\frac{\rho_{12} a_{2} / M}{\lambda_{2}+\rho_{21} / S}$

If we insert these two equations into system 12 (Eq. 12), we obtain a new system of two linear equations with two variables $a_{1}$ and $a_{2}$ :

$$
\begin{gathered}
\left\{\begin{array}{l}
m=a_{1} \exp \left(\lambda_{1} t\right)+a_{2} \exp \left(\lambda_{2} t\right) \\
s=\frac{\rho_{12} a_{1} / M}{\lambda_{1}+\rho_{21} / s} \exp \left(\lambda_{1} t\right)+\frac{\rho_{12} a_{2} / M}{\lambda_{2}+\rho_{21} / s} \exp \left(\lambda_{2} t\right)
\end{array}\right. \\
\Rightarrow m+s=a_{1} \exp \left(\lambda_{1} t\right)\left(1+\frac{\rho_{12} / M}{\lambda_{1}+\rho_{21} / S}\right) \\
+a_{2} \exp \left(\lambda_{2} t\right)\left(1+\frac{\rho_{12} / M}{\lambda_{2}+\rho_{21} / S}\right)
\end{gathered}
$$

Under initial conditions, system 13 (Eq. 13) becomes:

$\left\{\begin{array}{l}m_{0}=a_{1}+a_{2} \Leftrightarrow a_{1}=m_{0}-a_{2} \\ s_{0}=\frac{\rho_{12} a_{1} / M}{\lambda_{1}+\rho_{21} / s}+\frac{\rho_{12} a_{2} / M}{\lambda_{2}+\rho_{21} / s}\end{array}\right.$

Thus,

$$
\begin{aligned}
& s_{0}=\frac{\rho_{12}}{M}\left(\frac{m_{0}-a_{2}}{\lambda_{1}+\rho_{21} / S}+\frac{a_{2}}{\lambda_{2}+\rho_{21} / s}\right) \\
& \Leftrightarrow a_{2}=\left[\frac{\lambda_{1}+\rho_{21} / S}{\rho_{12} / M} s_{0}-m_{0}\right] \frac{\lambda_{2}+\rho_{21} / S}{\lambda_{1}-\lambda_{2}}
\end{aligned}
$$

\section{Appendix B}

Demonstration of equations modelling evolution over time of radioactivity in unlabelled Daphnia feeding on labelled algae

The kinetics of the tracer in each pool is described by (see Table 1 for explanation of symbols):

$\left\{\begin{aligned} \frac{d(m)}{d t} & =\frac{\rho_{21}}{S} s-\frac{\rho_{12}}{M} m-\frac{\rho_{10}}{M} m+\frac{\rho_{01}}{100} W S A \\ \frac{d(s)}{d t} & =\frac{\rho_{12}}{M} m-\frac{\rho_{21}}{S} s\end{aligned}\right.$

Let us define

$m_{t}=o_{t}+\alpha$

and

$s_{t}=u_{t}+\beta$

that we insert in the system of differential equations: 
$\left\{\begin{array}{l}\frac{d(o)}{d t}=-\frac{\left(\rho_{12}+\rho_{10}\right)}{M} o+\frac{\rho_{21}}{S} u-\frac{\left(\rho_{12}+\rho_{10}\right)}{M} \alpha+\frac{\rho_{21}}{S} \beta+\frac{\rho_{01}}{100} W S A \\ \frac{d(u)}{d t}=\frac{\rho_{12}}{M} o-\frac{\rho_{21}}{S} u+\frac{\rho_{12}}{M} \alpha-\frac{\rho_{21}}{S} \beta\end{array}\right.$

We can now determine $\alpha$ et $\beta$ which nullify the nonhomogeneous terms:

$$
\begin{aligned}
& \left\{\begin{array}{l}
\frac{\left(\rho_{12}+\rho_{10}\right)}{M} \alpha-\frac{\rho_{21}}{S} \beta=\frac{\rho_{01}}{100} W S A \\
\frac{\rho_{12}}{M} \alpha-\frac{\rho_{21}}{S} \beta=0
\end{array}\right. \\
& \Leftrightarrow\left\{\begin{array}{l}
\alpha=\frac{\rho_{01}}{\rho_{10} 100} W M S A \\
\beta=\frac{\rho_{12} \rho_{01}}{\rho_{10} \rho_{21} 100} W S S A
\end{array}\right.
\end{aligned}
$$

Both equations of system 17 (Eq. 17) become homogeneous:

$$
\left\{\begin{array}{l}
\frac{d(o)}{d t}=-\frac{\left(\rho_{12}+\rho_{10}\right)}{M} o+\frac{\rho_{21}}{S} u \\
\frac{d(u)}{d t}=\frac{\rho_{12}}{M} o-\frac{\rho_{21}}{S} u
\end{array}\right.
$$

As for the problem defined in Appendix A, we know that the solution of this linear differential system is a linear combination:

$$
\left\{\begin{array}{l}
o_{t}=a_{1} \exp \left(\lambda_{1} t\right)+a_{2} \exp \left(\lambda_{2} t\right) \\
u_{t}=b_{1} \exp \left(\lambda_{1} t\right)+b_{2} \exp \left(\lambda_{2} t\right)
\end{array}\right.
$$

where, as in Appendix $\mathrm{A}, b_{1}$ and $b_{2}$ are defined, respectively, by $b_{1}=\frac{\rho_{12} a_{1} / M}{\lambda_{1}+\rho_{21} / s}$, and $b_{2}=\frac{\rho_{12} a_{2} / M}{\lambda_{2}+\rho_{21} / s}$, and $\lambda_{1}$ and $\lambda_{2}$, respectively, by Eqs. 10 and 11 .

Under initial conditions, $m_{0}=0$ and $s_{0}=0$.

The insertion of Eqs. 15 and 16 into system 18 (Eq. 18) gives:

$\left\{\begin{array}{l}-\alpha=a_{1}+a_{2} \Leftrightarrow a_{1}=-\left(a_{2}+\alpha\right) \\ -\beta=b_{1}+b_{2}\end{array}\right.$

Thus,

$$
\begin{aligned}
-\beta & =\frac{\rho_{12}}{M}\left[\frac{-\left(a_{2}+\alpha\right)}{\lambda_{1}+\rho_{21} / S}+\frac{a_{2}}{\lambda_{2}+\rho_{21} / s}\right] \\
\Leftrightarrow a_{2} & =\left(\alpha-\frac{\lambda_{1}+\rho_{21} / S}{\rho_{12} / M} \beta\right) \frac{\lambda_{2}+\rho_{21} / S}{\lambda_{1}-\lambda_{2}}
\end{aligned}
$$

\section{References}

Andersen T, Hessen DO (1991) Carbon, nitrogen, and phosphorus content of freshwater zooplankton. Limnol Oceanogr 36:807814

Båmstedt U (1986) Chemical composition and energy content. In: Corner EDS, O'Hara (eds) The biological chemistry of marine copepods. Oxford University Press, Oxford, pp 1-58

Biddanda B, Ogdahl M, Cotner J (2001) Dominance of bacterial metabolism in oligotrophic relative to eutrophic waters. Limnol Oceanogr 46:730-739

Billen G (1984) Heterotrophic utilization and regeneration of nitrogen. In: Hobbie JE, Williams PJleB (eds) Heterotrophic activity in the sea. Plenum Press, New York, pp 313-355

Cimbleris ACP, Kalff J (1998) Planktonic bacterial respiration as a function of $\mathrm{C}: \mathrm{N}: \mathrm{P}$ ratios accross temperate lakes. Hydrobiologia 384:89-100
Conover RJ, Francis V (1973) The use of radioactive isotopes to measure the transfer of materials in aquatic food chains. Mar Biol 18:272-283

Copping AE, Lorenzen CJ (1980) Carbon budget of a marine phytoplankton-herbivore system with carbon-14 as a tracer. Limnol Oceanogr 25:873-882

DeMott WR, Gulati RD, Siewertsen K (1998) Effects of phosphorus-deficient diets on the carbon and phosphorus balance of Daphnia magna. Limnol Oceanogr 43:1147-1161

Elser JJ, Hassett RP (1994) A stoichiometric analysis of the zooplankton-phytoplankton interaction in marine and freshwater ecosystems. Nature 370:211-213

Elser JJ, Dobberfuhl DR, MacKay NA, Schampel JH (1996) Organism size, life history, and N:P stoichiometry: towards a unified view of cellular and ecosystem processes. BioScience 46:674-684

Elser JJ, Fagan WF, Denno RF, Dobberfuhl DR, Folarin A, Huberty A, Interlandi S, Kilham SS, McCauley E, Schulz KL, Siemann EH, Sterner RW (2000) Nutritional constraints in terrestrial and freshwater food webs. Nature 408:578-580

Gardner WS, Paffenhöfer G-A (1982) Nitrogen regeneration by the subtropical marine copepod Eucalanus pileatus. J Plankton Res 4:725-734

Goldman JC, Caron DA, Dennett MR (1987) Regulation of gross growth efficiency and ammonium regeneration in bacteria by substrate C:N ratio. Limnol Oceanogr 32:1239-1252

Goulden CE, Place AR (1990) Fatty acid synthesis and accumulation rates in daphniids. J Exp Zool 256:168-178

Groeger AW, Schram MD, Marzolf GR (1991) Influence of food quality on growth and reproduction in Daphnia. Freshwater Biol 26:11-19

Harder W, Dijkhuizen L (1983) Physiological responses to nutrient limitation. Annu Rev Microbiol 37:1-23

Hessen DO (1992) Nutrient element limitation of zooplankton production. Am Nat 140:799-814

Hessen DO (1997) Stoichiometry in food webs-Lotka revisited. Oikos 79:195-200

Hessen DO, Faafeng BA (2000) Elemental ratios in freshwater seston; implications for community structure and energy transfer in food webs. Arch Hydrobiol Adv Limnol 55:349-363

Hessen DO, Faerøvig PJ, Andersen T (2002) Light, nutrients, and $\mathrm{P}: \mathrm{C}$ ratios in algae: grazer performance related to food quality and food quantity. Ecology 83:1886-1898

Hopkinson CS, Sherr B, Wiebe WJ (1989) Size-fractionated metabolism of coastal microbial plankton. Mar Ecol Prog Ser 51:155-166

Hygum BH, Petersen JW, Søndergaard M (1997) Dissolved organic carbon released by zooplankton grazing activity - a highquality substrate pool for bacteria. J Plankton Res 19:97-111

Jumars PA, Penry DL, Baross JA, Perry MJ, Frost BW (1989) Closing the microbial loop: dissolved carbon pathway to heterotrophic bacteria from incomplete ingestion, digestion and absorption in animals. Deep-Sea Res PT A 36:483-495

Kilham SS, Kreeger DA, Lynn SG, Goulden CE, Herrera L (1998) COMBO: a defined freshwater culture medium for algae and zooplankton. Hydrobiologia 377:147-159

Lampert W (1975) A tracer study on the carbon turnover of Daphnia pulex. Verh Int Verein Limnol 19:2913-2921

Lampert W (1977) Studies on the carbon balance of Daphnia pulex as related to environmental conditions. I. Methodological problems of the use of ${ }^{14} \mathrm{C}$ for the measurement of carbon assimilation. Arch Hydrobiol [Suppl] 48:287-309

Lampert W (1978) Release of dissolved organic matter by grazing zooplankton. Limnol Oceanogr 23:831-834

Lampert W (1987) Feeding and nutrition in Daphnia. Mem Ist Ital Idrobiol Dott Marco de Marchi Pallanza Italy 45:143-192 
Lampert W, Bohrer R (1984) Effect of food availability on the respiratory quotient of Daphnia magna. Comp Biochem Phys A 78:221-223

Lampert W, Gabriel W (1984) Tracer kinetics in Daphnia: an improved two-compartment model and experimental test. Arch Hydrobiol 100:1-20

Lancelot C, Billen G (1985) Carbon-nitrogen relationships in nutrient metabolism of coastal marine ecosystems. Adv Aquat Microbiol 3:263-321

Olsen Y, Varum KM, Jensen A (1986) Some characteristics of the carbon compounds released by Daphnia. J Plankton Res 8:505517

Park J-Ch, Aizaki M, Fukushima T, Otsuki A (1997) Production of labile and refractory dissolved organic carbon by zooplankton excretion: an experimental study using large outdoor continuous flow-through ponds. Can J Fish Aquat Sci 54:434-443

Plath K, Boersma M (2001) Mineral limitation of zooplankton: stoichiometric constraints and optimal foraging. Ecology 82:1260-1269

Reynolds CS (1997) Vegetation processes in the pelagic: a model for ecosystem theory. In: Kinne O (ed) Excellence in ecology, vol 9. Ecology Institute, Oldendorf/Luhe, Germany

Richardot M, Debroas D, Thouvenot A, Sargos D, Dévaux J (2001) Influence of cladoceran grazing activity on dissolved organic matter, enzymatic hydrolysis and bacterial growth. J Plankton Res 23:1249-1261

Rosenstock B, Simon M (2001) Sources and sinks of dissolved free amino acids and protein in a large and deep mesotrophic lake. Limnol Oceanogr 46:644-654

Rothhaupt KO (1995) Algal nutrient limitation affects rotifer growth rate but not ingestion rate. Limnol Oceanogr 40:12011208

Salisbury FB, Ross CW (1992) Plant physiology, 4th edn. Wadsmorth Belmont, Calif.

Sterner RW (1993) Daphnia growth on varying quality of Scenedesmus: mineral limitation of zooplankton. Ecology 74:2351-2360
Sterner RW (1997) Modelling interactions of food quality and quantity in homeostatic consumers. Freshwater Biol 38:473481

Sterner RW (1998) Demography of a natural population of Daphnia retrocurva in a lake with low food quality. J Plankton Res 20:471-489

Sterner RW, Hessen DO (1994) Algal nutrient limitation and the nutrition of aquatic herbivores. Annu Rev Ecol Syst 25:1-29

Sterner RW, Smith RF (1993) Clearance, ingestion and release of N and P by Daphnia obtusa feeding on Scenedesmus acutus of varying quality. Bull Mar Sci 53:228-239

Sterner RW, Hagemeier DD, Smith RF, Smith WL (1992) Lipidovary indices in food-limited Daphnia. J Plankton Res 14:1449-1460

Sterner RW, Hagemeier DD, Smith WL, Smith RF (1993) Phytoplankton nutrient limitation and food quality for Daphnia. Limnol Oceanogr 38:857-871

Sterner RW, Clasen J, Lampert W, Weisse T (1998) Carbon:phosphorus stoichiometry and food chain production. Ecol Lett $1: 146-150$

Strom SL, Benner R, Ziegler S, Dagg MJ (1997) Planktonic grazers are a potentially important source of marine dissolved organic carbon. Limnol Oceanogr 42:1364-1374

Tessier AJ, Goulden CE (1982) Estimating food limitation in cladoceran populations. Limnol Oceanogr 27:707-717

Urabe J, Watanabe Y (1992) Possibility of N or P limitation for planktonic cladocerans: an experimental test. Limnol Oceanogr 37:244-251

Urabe J, Clasen J, Sterner RW (1997) Phosphorus limitation of Daphnia growth: Is it real? Limnol Oceanogr 42:1436-1443

Van Donk E, Hessen DO (1993) Grazing resistance in nutrientstressed phytoplankton. Oecologia 93:508-511

Vrede T, Andersen T, Hessen DO (1999) Phosphorus distribution in three crustacean zooplankton species. Limnol Oceanogr $44: 225-229$ 